\title{
Insulin resistance is an independent predictor of erectile dysfunction in patients with gout
}

\author{
Ji Hun Kim, Min Kyung Chung, Jin Young Kang, Jung Hee Koh, Jennifer Lee, Seung-Ki Kwok, \\ Ji Hyeon Ju, and Sung-Hwan Park
}

Division of Rheumatology, Department of Internal Medicine, College of Medicine, Seoul St. Mary's Hospital, The Catholic University of Korea, Seoul, Korea

Received: October 31, 2016 Revised : November 29, 2016 Accepted: November 30, 2016

\author{
Correspondence to \\ Sung-Hwan Park, M.D. \\ Division of Rheumatology, De- \\ partment of Internal Medicine, \\ College of Medicine, Seoul St. \\ Mary's Hospital, The Catholic \\ University of Korea, 222 Banpo- \\ daero, Seocho-gu, Seoul 06591, \\ Korea \\ Tel: +82-2-2258-6011 \\ Fax: +82-2-599-3589 \\ E-mail: rapark@catholic.ac.kr
}

Background/Aims: Gout is associated with metabolic disorders that are important risk factors for cardiovascular disease and erectile dysfunction (ED). We aimed to identify independent predictors of ED in patients with gout.

Methods: From August 2014 to August 2015, male outpatients who were being treated for gout in our rheumatology clinic and healthy males without any history of inflammatory disease (control group) were studied. ED was assessed in participants using the five-item version of the International Index of Erectile Function questionnaire. Insulin resistance (IR) was estimated using the homeostatic model assessment (HOMA-IR). Logistic regression analysis was performed to determine the effect of variables on ED risk in all of the study subjects and in patients with gout.

Results: We analyzed 80 patients with gout and 70 healthy controls. The median age of patients with gout was 52 years and median disease duration was 120 months. Gout patients were more likely to have ED than controls ( $55.3 \%$ vs. $41.4 \%$, $p<0.047$ ). After adjustment for confounding factors, only HOMA-IR was significantly associated with ED (odds ratio [OR], 1.82; 95\% confidence interval [CI], 1.05 to 3.15). Gout patients with ED were more likely to be older $(p<0.001)$, have higher HOMA-IR $(p=0.048)$, and have lower glomerular filtration rate $(p=0.038)$ than those without ED. Multivariate logistic regression analysis showed that HOMAIR was an independent predictor for ED (OR, 1.62; 95\% CI, 1.03 to 2.82) in gout patients.

Conclusions: IR is an independent predictor of ED in patients with gout.

Keywords: Arthritis, gouty; Erectile dysfunction; Insulin resistance

\section{INTRODUCTION}

Gout is the most common type of inflammatory arthritis in men and is characterized by deposition of monosodium urate crystals in joints and adjacent tissues, alongside chronic hyperuricemia $[1,2]$. However, gout is not just a joint disease but occurs in association with a number of metabolic disorders, including hypertension (HTN), obesity, diabetes mellitus (DM), dyslipidemia, chronic kidney disease, and metabolic syndrome [3-6]. Metabolic abnormalities are important risk factors for cardiovascular disease (CVD), which contributes to the increased mortality observed in patients with gout, as well as to the hyperuricemia and arthritic disease itself $[7,8]$. Consequently, previous guidelines have suggested that gout patients be assessed for comorbidities, including components of the metabolic syndrome, which should be treated as part of the management of gout $[9,10]$. 
Erectile dysfunction (ED) refers to a persistent inability to achieve and maintain an erection sufficient to complete sexual intercourse [11] and is common in the general population. ED reflects an early stage of vascular pathology, specifically relating to endothelial dysfunction [12]. Many pathophysiologic mechanisms have been identified that contribute to the development of ED, many of which are also comorbidities of gout. These overlap not only with conventional risk factors for CVD, such as aging, HTN, DM, chronic kidney disease, and dyslipidemia, but also with obesity-induced hypogonadism, insulin resistance (IR), and low grade inflammation [13-16]. Although previous studies have already reported an increased risk of ED in gout patients [17-19], most of these studies were designed to identify the prevalence and association between gout and ED compared to a control group, and not to evaluate clinical and laboratory features of metabolic disorders in gout patients.

In this study, we have investigated the association between the metabolic disorders of gout and ED. In addition, we have sought to identify which metabolic variables could represent independent risk factors for the presence of ED in gouty arthritis.

\section{METHODS}

\section{Patients and controls}

We enrolled 102 male outpatients who were being treated for gout at a tertiary care hospital between August 2014 and August 2015. The diagnosis of gout was based on a history of the identification of monosodium urate crystals in affected joint fluid or fulfillment of the clinical criteria of the American Rheumatism Association for primary gout, as determined by rheumatologists [20]. We only included patients who had not suffered an attack of gout in the 6 months preceding enrollment. Eighty-seven healthy males without any history of inflammatory disease were also recruited as a control group. For the analysis of data, we selected individuals aged between 35 and 70 years and excluded subjects with histories of coronary artery disease, cerebrovascular events, peripheral arterial disease, DM, or benign prostatic hyperplasia (BPH). The study was approved by the Institutional Review Board of Seoul St. Mary's Hospital (KC14QISIo626). All subjects gave their informed consent.

\section{Baseline characteristics and laboratory findings}

Age, height, weight, and waist circumference were measured in all subjects. Body mass index (BMI) was calculated in $\mathrm{kg} / \mathrm{m}^{2}$. The subjects' history of alcohol consumption, smoking, and the average number of hours of exercise undertaken per week were recorded. The details of comorbidities in subjects with HTN or dyslipidemia who had been prescribed treatment were also recorded, along with disease duration and the type of gout medication being used (colchicine, allopurinol, benzbromarone, or febuxostat) by gout patients. Subjects were required to complete the Hospital Anxiety and Depression Scale (HADS) questionnaire, which is designed to assess anxiety and depression as an evaluation of mood status. This incorporates seven measures on each subscale that are independent of somatic symptoms [21].

Overnight fasting blood samples were obtained for the determination of hemoglobin, platelet count, creatinine, uric acid, glucose, insulin, testosterone, $25(\mathrm{OH})$-vitamin D, total cholesterol, triglyceride (TG), high density lipoprotein cholesterol, and low density lipoprotein cholesterol. Erythrocyte sedimentation rate (ESR) and C-reactive protein (CRP) were determined only in gout patients. Glomerular filtration rate (GFR) was calculated using the modification of diet in renal disease (MDRD) formula (MDRD-GFR $=186 \times$ serum creatinine $[\mathrm{mg} / \mathrm{dL}$ ] ${ }^{-1.154} \times$ age $\left.^{-0.203}\right)$. IR was evaluated using the homeostatic model assessment (HOMA-IR): HOMA-IR = insulin $(\mu \mathrm{IU} / \mathrm{mL}) \times$ glucose $(\mathrm{mmol} / \mathrm{L}) / 22.5$.

\section{Assessment of erectile function}

ED was assessed using the five-item version of the International Index of Erectile Function (IIEF-5) and this was validated using a self-administered questionnaire [22]. Each question is scored from 1 to 5, with a maximum score of 25 denoting perfect erectile function. The severity of ED is generally classified into five categories: severe ( 1 to 7 ), moderate ( 8 to 11 ), mild to moderate ( 12 to 16), mild (17 to 21), and normal (22 to 25). The presence of ED was defined by an IIEF-5 score of below the normal range.

\section{Statistical analysis}

Continuous variables are expressed as median (interquartile range $[\mathrm{IQR}]$ ) and categorical variables as absolute numbers (\%). We compared the variables between 
patients with gout and the control group, as well as between the groups of gout patients with and without ED, using the Mann-Whitney Utest for continuous variables, and the chi-square or Fisher exact tests for categorical variables. Logistic regression analysis was performed to identify which variables were independent risk factors for the development of ED, involving adjustment for important confounders. A $p<0.05$ was considered to be statistically significant. All data were analyzed using the SPSS version 22.0 (IBM Corp., Armonk, NY, USA).

\section{RESULTS}

\section{Demographics and clinical characteristics of subjects}

Eighty gout patients and 70 control subjects were included, and 22 patients with gout and 11 control subjects were excluded. Of these excluded subjects, seven were aged less than 35 or over 70 among the gout patients and eight among the controls. The subjects excluded because of a history of comorbid disease comprised $15 \mathrm{pa}$ tients with gout (six CVD, five DM, and BPH) and nine in the control group (three DM and six BPH). Baseline demographic and clinical characteristics are presented in Table 1. The median age of patients with gout was slightly greater than the controls, but this difference did not reach statistical significance ( 52 years [IQR, 44 to 59] vs. 50 years [IQR, 42 to 55], $p=0.090$ ). Weight, BMI, and waist circumference were significantly higher in gout patient than in controls $(p=0.019, p<0.001$, and $p<$ 0.001 , respectively). There were no differences between the groups with regard to history of smoking, alcohol consumption, or exercise. Gout patients had a median systolic blood pressure of $124 \mathrm{mmHg}$ (IQR, 119 to 130) and diastolic blood pressure of $83 \mathrm{mmHg}$ (IQR, 78 to 87 ). The HADS questionnaires showed that both anxiety and depression scores were similar between the two groups.

There was no difference in fasting glucose between gout patients and controls. By contrast, the median plasma insulin and HOMA-IR were significantly higher in gout patients than controls (insulin: $8.02 \mu \mathrm{IU} / \mathrm{mL}$ [IQR, 6.01 to 11.38$]$ vs. $5.48 \mu \mathrm{IU} / \mathrm{mL}$ [IQR, 3.19 to 7.73$]$ in controls, $p$ < 0.001; HOMA-IR: $1.86 \mathrm{~mL} / \mathrm{min} / 1.73 \mathrm{~m}^{2}$ [IQR, 1.55 to 2.70] vs. $1.29 \mathrm{~mL} / \mathrm{min} / 1.73 \mathrm{~m}^{2}$ [IQR, 0.82 to 1.77 ] in con trols, $p<$ 0.001 ), as expected. Patients with gout had similar median serum uric acid to controls $(5.9 \mathrm{mg} / \mathrm{dL}$ [IQR, 5.0 to 6.7 ] vs. $5.9 \mathrm{mg} / \mathrm{dL}[\mathrm{IQR}, 5.0$ to 6.4$]$ in controls, $p=0.393$ ). The gout patients had significantly higher serum creatinine and lower GFR than controls. There were no significant differences in lipid profiles between groups, except that plasma TG was higher in the gout patients $(p=0.005)$. Plasma testosterone was significantly lower in gout patients than controls $(p<0.001)$, as was $25(\mathrm{OH})$-vitamin D $(p=0.045)$. In gout patients, the median ESR was 9 (IQR, 4.5 to 16 ) and CRP was 0.11 (IQR, 0.04 to 0.20 ). Gout patients had higher prevalences of the comorbidities HTN and dyslipidemia than control subjects (34.2\% vs. $8.6 \%$ in controls, $p<0.001 ; 77.6 \%$ vs. $2.9 \%$ in controls, $p<0.001$ ).

The median IIEF-5 score was lower in the patient group than the control group, but this did not reach statistical significance (21 [IQR, 17 to 24 ] vs. 22 [IQR, 18 to 24] in controls, $p=0.443$ ). However, the incidence of ED was significantly higher in the patient group than in the control group (55.3\% vs. $41.4 \%$ in control, $p=0.047$ ). In gout patients, the median disease duration was 120 months (IQR, 59 to 207). Of gout patients, 97.5\% were currently using urate-lowering medication. Allopurinol was most frequently used (61.3\%), followed by febuxostat (27.5\%), and benzbromarone (8.8\%).

\section{Associations between clinical variables and ED in study subjects}

We performed univariate and multivariate analysis of ED risk using variables that showed statistically significant differences between the groups (Table 2). Univariate analyses did not demonstrate a significant association of gout with $\mathrm{ED}$ (OR, 1.69; 95\% CI, 0.88 to 3.23). Older age, higher waist circumference, higher HOMA-IR, and lower MDRD-GFR and testosterone level were significant predictors of ED in unadjusted analyses. Comorbidities such as HTN and dyslipidemia were not significantly associated with ED in gout patients. Multivariate logistic regression analysis revealed that only HOMA-IR was significantly associated with ED (adjusted OR, 1.82; 95\% CI, 1.05 to 3.15$)$.

\section{Differences in clinical variables between gout pa- tients with and without ED}

To determine whether there were differences in clinical variables between gout patients with and without ED, patients were divided into two groups (44 patients with ED and 36 without). Variables that were significantly 
Table 1. Demographics and clinical characteristics of the study participants

\begin{tabular}{|c|c|c|c|}
\hline Characteristic & Patients with gout $(\mathrm{n}=80)$ & Controls $(\mathrm{n}=70)$ & $p$ value \\
\hline Age, yr & $52(44-59)$ & $50(42-55)$ & 0.090 \\
\hline Height, cm & $172(167-176)$ & $172(168-176)$ & 0.813 \\
\hline Weight, kg & $74(68-81)$ & $71(65-75)$ & 0.019 \\
\hline Body mass index, $\mathrm{kg} / \mathrm{m}^{2}$ & $25 \cdot 3(23.6-27.1)$ & $24.0(22.3-25.0)$ & $<0.001$ \\
\hline Waist circumference, $\mathrm{cm}$ & $86.8(83.8-92.4)$ & $83.0(79.0-86.3)$ & $<0.001$ \\
\hline History of smoking & $57(75.0)$ & $45(64 \cdot 3)$ & 0.091 \\
\hline Current smoking & $29(38.2)$ & $35(50.0)$ & 0.116 \\
\hline Smoking, pack/year & $17(10-30)$ & $16(10-23)$ & 0.412 \\
\hline Alcohol drinking, no./wk & $1(0-3)$ & $2(1-3)$ & 0.126 \\
\hline Exercise, no./wk & $3(1-5)$ & $2(1-4)$ & 0.595 \\
\hline \multicolumn{4}{|l|}{ HASD score } \\
\hline Anxiety score & $4(2-6)$ & $5(2-7)$ & 0.153 \\
\hline Depression score & $4(2-7)$ & $5(3-6)$ & 0.542 \\
\hline Hemoglobin, g/dL & $15 \cdot 3(14.5-16.0)$ & $15.6(14.7-16.5)$ & 0.277 \\
\hline Platelet, $10^{9} / \mathrm{L}$ & $218(192-260)$ & $233(212-265)$ & 0.050 \\
\hline Fasting glucose, mg/dL & $99(94-105)$ & $95(89-102)$ & 0.117 \\
\hline Insulin, $\mu \mathrm{IU} / \mathrm{mL}$ & $8.02(6.01-11.38)$ & $5.48(3.19-7.73)$ & $<0.001$ \\
\hline HOMA-IR & $1.86\left(1.55^{-2.70}\right)$ & $1.29(0.82-1.77)$ & $<0.001$ \\
\hline Uric acid, mg/dL & $5.9(5.0-6.7)$ & $5.9(5.0-6.4)$ & 0.393 \\
\hline BUN, mg/dL & $15.2(12.7-17.2)$ & $14.1(12.2-18.2)$ & 0.630 \\
\hline Creatinine, mg/dL & $1.07(0.96-1.22)$ & $0.95(0.89-1.03)$ & $<0.001$ \\
\hline MDRD-GFR, $\mathrm{mL} / \mathrm{min} / 1.73 \mathrm{~m}^{2}$ & $83.3(66.8-102.2)$ & $96.1(88.1-111.7)$ & 0.006 \\
\hline Total cholesterol, mg/dL & $180(162-206)$ & $176(165-200)$ & 0.629 \\
\hline Triglyceride, mg/dL & $184(113-238)$ & $138(84-160)$ & 0.005 \\
\hline $\mathrm{LDL}, \mathrm{mg} / \mathrm{dL}$ & $105(84-125)$ & $109(92-122)$ & 0.703 \\
\hline $\mathrm{HDL}, \mathrm{mg} / \mathrm{dL}$ & $47(41-52)$ & $46(42-57)$ & 0.564 \\
\hline Testosterone, ng/mL & $3.92(3.34-5.10)$ & $5.32(4.66-6.39)$ & $<0.001$ \\
\hline${ }_{25}(\mathrm{OH})$ vitamin $\mathrm{D}, \mathrm{ng} / \mathrm{mL}$ & $23.37(17.93-27.96)$ & $26(22.09-29.13)$ & 0.045 \\
\hline \multicolumn{4}{|l|}{ Comorbidities } \\
\hline Hypertension & $26(34.2)$ & $6(8.6)$ & $<0.001$ \\
\hline Dyslipidemia & $59(77.6)$ & $2(2.9)$ & $<0.001$ \\
\hline \multicolumn{4}{|l|}{ Erectile function } \\
\hline Total IIEF-5 score & $21(17-24)$ & $22(18-24)$ & 0.443 \\
\hline Erectile dysfunction & $42(55 \cdot 3)$ & $29(41.4)$ & 0.047 \\
\hline Gout disease duration, mon & $120(59-207)$ & & \\
\hline \multicolumn{4}{|l|}{ Medication of gout } \\
\hline Colchicine & $22(27.5)$ & & \\
\hline Allopurinol & $49(61.3)$ & & \\
\hline Febuxostat & $22(27 \cdot 5)$ & & \\
\hline Benzbromarone & $7(8.8)$ & & \\
\hline
\end{tabular}

Values are presented as median (interquartile range) or number (\%).

HADS, hospital anxiety and depression scale; HOMA-IR, homeostatic model assessment of insulin resistance; BUN, blood urea nitrogen; MDRD-GFR, modification of diet in renal disease-glomerular filtration rate; LDL, low density lipoprotein; HDL, high density lipoprotein; IIEF-5, 5-item version of the International Index of Erectile Function. 
different between groups and important confounders are presented in Table 3. Gout patients with ED were significantly older and had higher HOMA-IR and lower MDRD-GFR than patients without ED $(p<0.001, p=$ 0.048 , and $p=0.038$, respectively). Subgroup analysis of gout patients with data from 6 months check-ups for 2 years prior to the study showed no significant differences of ED between those who had ever changed in serum uric acid over $6 \mathrm{mg} / \mathrm{dL}$ and had not (data not shown).

\section{Independent predictors of ED in gout patients}

Age (OR, 1.12; 95\% CI, 1.05 to 1.19) and HOMA-IR (OR, 1.57; $95 \% \mathrm{CI}, 1.14$ to 2.60 ) were identified as risk factors

Table 2. Unadjusted and adjusted ORs for erectile dysfunction

\begin{tabular}{lcc}
\hline Variable & $\begin{array}{c}\text { Unadjusted OR } \\
(95 \% \mathrm{CI})\end{array}$ & $\begin{array}{c}\text { Adjusted OR } \\
(95 \% \mathrm{CI})^{\mathrm{a}}\end{array}$ \\
\hline Gout & $1.69(0.88-3.23)$ & $0.62(0.11-3.53)$ \\
Age & $1.11(1.06-1.17)$ & $1.09(0.99-1.20)$ \\
Waist circumference & $1.07(1.01-1.13)$ & $1.04(0.92-1.17)$ \\
HOMA-IR & $1.71(1.11-2.64)$ & $1.82(1.05-3.15)$ \\
MDRD-GFR & $0.98(0.96-0.99)$ & $0.99(0.96-1.03)$ \\
Testosterone & $0.72(0.53-0.97)$ & $0.99(0.68-1.46)$ \\
HTN & $1.61(0.74-3.53)$ & $1.48(0.36-5.65)$ \\
Dyslipidemia & $1.37(0.71-2.64)$ & $0.54(0.17-1.75)$ \\
\hline
\end{tabular}

OR, odds ratio; CI, confidence interval; HOMA-IR, homeostatic model assessment of insulin resistance; MDRD-GFR, modification of diet in renal disease-glomerular filtration rate; HTN, hypertension.

${ }^{a}$ Adjusted ORs for the participants were adjusted for gout, age, waist circumference, HOMA-IR, MDRD-GFR, testosterone, HTN, and dyslipidemia. for ED in gout patients using univariate analyses (Table 4). To identify independent predictors of ED in gout patients, multivariate logistic regression was performed. Age, waist circumference, HOMA-IR, MDRD-GFR, testosterone level, and history of HTN or dyslipidemia were entered into a logistic regression model as independent confounders of ED risk. After adjustment for covariates, HOMA-IR (adjusted OR, 1.62; 95\% CI, 1.03 to 2.82) was shown to be an independent predictor of ED in gout patients.

\section{DISCUSSION}

In this study, we have observed a significantly increased prevalence of ED in patients with gout vs. healthy controls. We have also shown that gout patients were significantly more likely to have metabolic abnormalities, such as obesity, increased HOMA-IR, reduced renal function, HTN, and dyslipidemia. However, only HOMA-IR showed a strong significant association with ED after multivariate analysis. In addition, HOMA-IR was an independent risk factor for ED in gout patients after adjustment for confounding variables.

Recently, Chen et al. [17] showed that there is a higher incidence of ED in gout patients than in subjects without gout (12.36 per 10,000 person-years vs. 9.07 per 10,000 person-years, $p<0.001$ ), and a 1.21-fold adjusted hazard ratio for ED in gout versus the control group in their cohort study. Our results have also shown that there is an approximately 1.3-fold higher prevalence of ED in gout patients compared to controls. Schlesinger et al. [19] reported that a significantly higher proportion

Table 3. Comparison of gout patients with and without ED

\begin{tabular}{lccc}
\hline Characteristics & With ED $(\mathrm{n}=44)$ & Without ED $(\mathrm{n}=36)$ & $p$ value \\
\hline Age, $\mathrm{yr}$ & $55(50-60)$ & $48(41-55)$ & $<0.001$ \\
Waist circumference, $\mathrm{cm}$ & $87.0(83.9-93.9)$ & $86.0(81.3-90.4)$ & 0.316 \\
Insulin, $\mu \mathrm{IU} / \mathrm{mL}$ & $8.82(6.89-12.65)$ & $7.28(4.86-9.28)$ & 0.061 \\
HOMA-IR & $2.05(1.72-3.05)$ & $1.75(1.06-2.35)$ & 0.048 \\
Creatinine, $\mathrm{mg} / \mathrm{dL}$ & $1.09(0.99-1.22)$ & $1.05(0.91-1.22)$ & 0.484 \\
MDRD-GFR, $\mathrm{mL} / \mathrm{min} / 1.73 \mathrm{~m}^{2}$ & $77.0(64.8-96.4)$ & $94.9(69.9-108.9)$ & 0.038 \\
Testosterone, $\mathrm{ng} / \mathrm{mL}$ & $3.82(3.25-4.78)$ & $4.10(3.76-5.23)$ & 0.100 \\
\hline
\end{tabular}

ED, erectile dysfunction; HOMA-IR, homeostatic model assessment of insulin resistance; MDRD-GFR, modification of diet in renal disease-glomerular filtration rate. 
Table 4. Unadjusted and adjusted OR for erectile dysfunction in gout

\begin{tabular}{lcc}
\hline Variable & $\begin{array}{c}\text { Unadjusted OR } \\
(95 \% \mathrm{CI})\end{array}$ & $\begin{array}{c}\text { Adjusted OR } \\
(95 \% \mathrm{CI})^{\mathrm{a}}\end{array}$ \\
\hline Age & $1.12(1.05-1.19)$ & $1.08(0.97-1.21)$ \\
HOMA-IR & $1.57(1.14-2.60)$ & $1.62(1.03-2.82)$ \\
MDRD-GFR & $0.99(0.97-1.01)$ & $0.99(0.96-1.04)$ \\
\hline
\end{tabular}

OR, odds ratio; CI, confidence interval; HOMA-IR, homeostatic model assessment of insulin resistance; MDRD-GFR, modification of diet in renal disease-glomerular filtration rate.

${ }^{a}$ Adjusted ORs for the patients were adjusted for the covariates age, waist circumference, HOMA-IR, MDRD-GFR, testosterone, hypertension, and dyslipidemia.

of gout patients also had ED, when compared to subjects without gout but with other rheumatic diseases, including rheumatoid arthritis, osteoarthritis, and psoriatic arthritis ( $76 \%$ vs. $51 \%, p=0.0003)$. The prevalence of ED in gout patients in this study was approximately $20 \%$ higher than in our study, which may be explained by the exclusion from our analysis of participants with CVD, $\mathrm{DM}$, or BPH, and those aged less than 35 or over 70 .

Mounting evidence suggests that both hyperuricemia and inflammation can increase the risk of ED, especially in patients with gout. Salem et al. [23] reported that serum uric acid level was predictive of ED and several studies have shown an association between hyperuricemia and endothelial dysfunction, which causes ED by decreasing nitric oxide production and increasing production of reactive oxygen species [24]. Low grade inflammation, represented by increased levels of plasma markers, such as CRP, tumor necrosis factor- $\alpha$, and interleukin-6, is also positively associated with ED $[15,25]$.

The prevalence of IR in patients with gout has also been previously investigated. Yoo et al. [26] reported that gouty arthritis patients had significantly higher IR than healthy controls (HOMA-IR, $2.63 \pm 1.36$ vs. $1.91 \pm 1.01$, respectively). Another report estimated that hyperinsulinemia and IR occur in $95 \%$ and $76 \%$ of gout patients, respectively [27]. Furthermore, increased plasma insulin in gout patients was independently correlated with hyperuricemia, which is also associated with coronary artery disease $[28,29]$. Insulin can increase renal reabsorption of urate through stimulation of the tubular ion exchange and elevate concentration of systemic adenos- ine, which leads to renal retention of urate [30]. Given that IR plays an important role in the pathogenesis of endothelial dysfunction and atherosclerosis by promoting a direct proatherogenic effect in the arterial wall, which can subsequently progress to plaque formation and coronary artery disease [31,32], we hypothesized that increased IR has an important role in the development of ED in gout patients. This hypothesis was supported by a previous study showing that endothelial dysfunction and atherosclerosis induced by IR and inflammation resulted in dysregulation of both the penile and coronary artery circulations [33]. Consistent with the hypothesis, we found that IR was a significant independent risk factor for ED in gout, after adjustment for confounding factors.

There are several limitations to our study. First, IIEF5 is a validated tool for the assessment of ED but may not accurately differentiate between organic and psychogenic ED [34,35]. Second, although anti-hypertensive drugs such as $\beta$-blockers, thiazides, and calcium channel blockers can affect both ED and IR [36,37], we did not make a detailed assessment of patient prescriptions. Third, all of the subjects had presented to our tertiary medical institution; therefore, there could have been a selection bias and extrapolation of the conclusions of the present study to all gout patients should be undertaken with caution. Despite the limitations, however, we believe that our study has important implications. This is the first study to reveal which metabolic parameter represents an independent risk factor for ED in patients with gout. Furthermore, to minimize the effects of organic risk factors for ED and gout itself, we excluded subjects with several comorbidities and histories of attacks of gout within the 6 months preceding their participation. The interesting finding of no difference in uric acid level between patients and controls and the low levels of ESR and CRP in gout patients might reflect these exclusion criteria. Thus, the present study is especially relevant to patients with clinically stable gout and without significant active inflammation.

In conclusion, we have confirmed that IR is an independent predictor of the presence of ED in patients with gout. Our finding implies that insulin sensitivity should be assessed to assist in the prevention of ED in patients with gout, even when disease activity and serum uric acid are well controlled. 


\section{KEY MESSAGE}

1. Patients with gout had a higher prevalence of erectile dysfunction (ED) than controls.

2. Risk factors of ED were older age, higher homeostatic model assessment of insulin resistance, and lower glomerular filtration rate in gout.

3. In our study, insulin resistance was an independent predictor of ED in patients with gout.

\section{Conflict of interest}

No potential conflict of interest relevant to this article was reported.

\section{REFERENCES}

1. Richette P, Bardin T. Gout. Lancet 2010;375:318-328.

2. Zhu Y, Pandya BJ, Choi HK. Prevalence of gout and hyperuricemia in the US general population: the National Health and Nutrition Examination Survey 2007-2008. Arthritis Rheum 2011;63:3136-3141.

3. Fessel WJ. Renal outcomes of gout and hyperuricemia. Am J Med 1979;67:74-82.

4. Takahashi S, Yamamoto T, Moriwaki Y, Tsutsumi Z, Higashino K. Increased concentrations of serum Lp(a) lipoprotein in patients with primary gout. Ann Rheum Dis 1995;54:90-93.

5. Grodzicki T, Palmer A, Bulpitt CJ. Incidence of diabetes and gout in hypertensive patients during 8 years of follow-up. The General Practice Hypertension Study Group. J Hum Hypertens 1997;11:583-585.

6. Choi HK, Ford ES, Li C, Curhan G. Prevalence of the metabolic syndrome in patients with gout: the Third National Health and Nutrition Examination Survey. Arthritis Rheum 2007:57:109-115.

7. Abbott RD, Brand FN, Kannel WB, Castelli WP. Gout and coronary heart disease: the Framingham Study. J Clin Epidemiol 1988;41:237-242.

8. Choi HK, Curhan G. Independent impact of gout on mortality and risk for coronary heart disease. Circulation 2007;116:894-900.

9. Zhang W, Doherty M, Bardin T, et al. EULAR evidence based recommendations for gout. Part II: Management.
Report of a task force of the EULAR Standing Committee for International Clinical Studies Including Therapeutics (ESCISIT). Ann Rheum Dis 2006;65:1312-1324.

10. Zhang W, Doherty M, Pascual E, et al. EULAR evidence based recommendations for gout. Part I: Diagnosis. Report of a task force of the Standing Committee for International Clinical Studies Including Therapeutics (ESCISIT). Ann Rheum Dis 2006;65:1301-1311.

11. Hatzimouratidis K, Amar E, Eardley I, et al. Guidelines on male sexual dysfunction: erectile dysfunction and premature ejaculation. Eur Urol 2010;57:804-814.

12. Kloner RA. Erectile dysfunction in the cardiac patient. Curr Urol Rep 2003;4:466-471.

13. Hermann GG, Horn T, Steven K. The influence of the level of lamina propria invasion and the prevalence of p53 nuclear accumulation on survival in stage $\mathrm{T} 1$ transitional cell bladder cancer. J Urol 1998;159:91-94.

14. Feldman HA, Johannes CB, Derby CA, et al. Erectile dysfunction and coronary risk factors: prospective results from the Massachusetts male aging study. Prev Med 2000;30:328-338.

15. Chiurlia E, D'Amico R, Ratti C, Granata AR, Romagnoli R, Modena MG. Subclinical coronary artery atherosclerosis in patients with erectile dysfunction. J Am Coll Cardiol 2005;46:1503-1506.

16. Heidler S, Temml C, Broessner C, et al. Is the metabolic syndrome an independent risk factor for erectile dysfunction? J Urol 2007;177:651-654.

17. Chen YF, Lin HH, Lu CC, et al. Gout and a subsequent increased risk of erectile dysfunction in men aged 64 and under: a nationwide cohort study in Taiwan. J Rheumatol 2015;42:1898-1905.

18. Hsu CY, Lin CL, Kao CH. Gout is associated with organic and psychogenic erectile dysfunction. Eur J Intern Med 2015;26:691-695.

19. Schlesinger N, Radvanski DC, Cheng JQ, Kostis JB. Erectile dysfunction is common among patients with gout. J Rheumatol 2015;42:1893-1897.

20. Wallace SL, Robinson H, Masi AT, Decker JL, McCarty DJ, Yu TF. Preliminary criteria for the classification of the acute arthritis of primary gout. Arthritis Rheum 1977;20:895-900.

21. Herrmann C. International experiences with the Hospital Anxiety and Depression Scale: a review of validation data and clinical results. J Psychosom Res 1997;42:17-41.

22. Rosen RC, Riley A, Wagner G, Osterloh IH, Kirkpatrick 
J, Mishra A. The international index of erectile function (IIEF): a multidimensional scale for assessment of erectile dysfunction. Urology 1997;49:822-830.

23. Salem S, Mehrsai A, Heydari R, Pourmand G. Serum uric acid as a risk predictor for erectile dysfunction. J Sex Med 2014;11:1118-1124.

24. Kanbay M, Segal M, Afsar B, Kang DH, Rodriguez-Iturbe B, Johnson RJ. The role of uric acid in the pathogenesis of human cardiovascular disease. Heart 2013;99:759-766.

25. Vlachopoulos C, Aznaouridis K, Ioakeimidis N, et al. Unfavourable endothelial and inflammatory state in erectile dysfunction patients with or without coronary artery disease. Eur Heart J 2006;27:2640-2648.

26. Yoo HG, Lee SI, Chae HJ, Park SJ, Lee YC, Yoo WH. Prevalence of insulin resistance and metabolic syndrome in patients with gouty arthritis. Rheumatol Int 2011;31:485491.

27. Dessein PH, Shipton EA, Stanwix AE, Joffe BI, Ramokgadi J. Beneficial effects of weight loss associated with moderate calorie/carbohydrate restriction, and increased proportional intake of protein and unsaturated fat on serum urate and lipoprotein levels in gout: a pilot study. Ann Rheum Dis 2000;59:539-543.

28. Lee J, Sparrow D, Vokonas PS, Landsberg L, Weiss ST. Uric acid and coronary heart disease risk: evidence for a role of uric acid in the obesity-insulin resistance syndrome. The Normative Aging Study. Am J Epidemiol 1995;142:288-294.

29. Rathmann W, Funkhouser E, Dyer AR, Roseman JM. Relations of hyperuricemia with the various components of the insulin resistance syndrome in young black and white adults: the CARDIA study. Coronary Artery Risk Development in Young Adults. Ann Epidemiol 1998;8:250261.

30. Choi HK, Mount DB, Reginato AM; American College of Physicians; American Physiological Society. Pathogenesis of gout. Ann Intern Med 2005;143:499-516.

31. Bornfeldt KE, Tabas I. Insulin resistance, hyperglycemia, and atherosclerosis. Cell Metab 2011;14:575-585.

32. Karrowni W, Li Y, Jones PG, et al. Insulin resistance is associated with significant clinical atherosclerosis in nondiabetic patients with acute myocardial infarction. Arterioscler Thromb Vasc Biol 2013;33:2245-2251.

33. Gandaglia G, Briganti A, Jackson G, et al. A systematic review of the association between erectile dysfunction and cardiovascular disease. Eur Urol 2014;65:968-978.

34. Blander DS, Sanchez-Ortiz RF, Broderick GA. Sex inventories: can questionnaires replace erectile dysfunction testing? Urology 1999;54:719-723.

35. Deveci S, O’Brien K, Ahmed A, Parker M, Guhring P, Mulhall JP. Can the International Index of Erectile Function distinguish between organic and psychogenic erectile function? BJU Int 2008;102:354-356.

36. Lithell HO. Effect of antihypertensive drugs on insulin, glucose, and lipid metabolism. Diabetes Care 1991;14:203209.

37. Lue TF. Erectile dysfunction. N Engl J Med 2000;342:18021813 . 